\title{
An Interesting Angle for Explaining Innovations of Biomaterial to College students-Drug Controlled Release Profiles
}

\author{
Ke Wang, Jiayi Huang, Xiaoyu Liu, Mingjie Zhu, Yangcao Deng, Deng-Guang Yu* \\ School of Materials Science and Engineering, University of Shanghai for Science and Technology \\ 516 Jungong Road, Shanghai 200093, China \\ ydg017@usst.edu.cn \\ *Corresponding author
}

\begin{abstract}
Innovation capability is one of the most important abilities that the college students should foster during their education period. Explaining the innovation to students clearly is the primary task and also a very important step in their study courses. Based on the medicated applications of drug-loaded biomaterials for providing different kinds of drug controlled release profiles, this paper shows an interesting angle for the teachers to achieve vivid teaching materials for the students majoring in material science and engineering. In addition to the polymer-based medicated materials, the materials with complex nanostructural characteristics, the advanced techniques and processes for creating these biomaterials (such as electrospinning and electrospraying) can also be fully and vividly explained to the students in a comprehensive and systematic manner. This study provides a new way for teachers in universities and colleges to obtain practicable teaching materials from their scientific researches and to carry out innovation lessons for undergraduate students.
\end{abstract}

Keywords-Teaching materials; Innovation education; College students; Biomaterials; Drug controlled release

\section{INTRODUCTION}

Today, innovation education is very important for fostering the students' comprehensive capabilities during their college life. However, one dilemma exists always in the higher education reformations. On one hand, all the people around the higher education hope that there are new atmosphere, under which the innovative teaching-learning circle can be built between the teachers and the students. But on the other hand, the traditional teaching materials, the complicated professional theories and the unchangeable management model makes the reformations very hard, and even impossible, to effectively carry out. The solution of this situation needs teachers' subjective initiative.

The modern science and engineering is developing in an accelerated manner. Most of the teachers in the university spend their time not only in teaching but also in scientific research. However, very small portion of them think enough about how to exploit their research topics as vivid innovation teaching materials for the undergraduate students. Based on this situation, here, we propose an interesting angle for explaining innovations of biomaterial to college students. This angle is based on our scientific studies about drug controlled release profiles, and their related nanomaterials and advanced nanotechnologies.

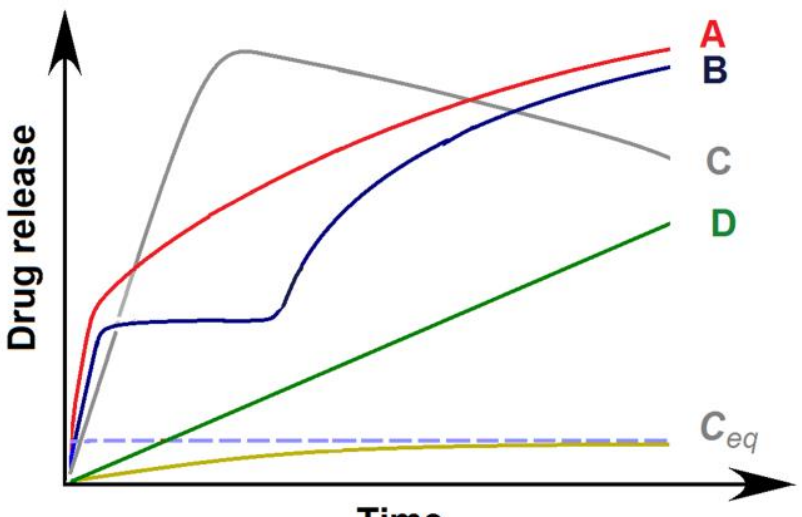

Time

Fig. 1 Different kinds of drug controlled release profiles: A-traditional sustained release with an initial burst release effect; B-a typical biphasic drug controlled release; $\mathrm{C}$-a pulsatile release; D-the idea zero-order linear release; $C_{\text {eq }}$ indicates the saturated solubility of a poorly water-soluble drug.

Shown in Fig. 1 is a series of drug controlled release profiles, including traditional sustained release with an initial burst release effect, typical biphasic drug controlled release, pulsatile release, and zero-order linear release. These typical drug controlled release profiles are popular in present pharmaceutics [1-6]. A wide variety of pharmaceutical excipients and advanced technologies have been explored for potential applications in providing these drug controlled release characteristics.

Among different types of advanced techniques, electrohydrodynamic atomization (EHDA) is one of the fastest developing processes. It has been divided into several categories, such as electrospinning, electrospraying, and e-jet printing. Electrospinning, derived from the word "electrostatic spinning", is a straightforward, one-step, "top-down" nanofiber fabrication process. It has shown great capability in generating all kinds of functional nanofibers. A series of unique properties have made it a very popular tool in the present scientific research fields and also in the potential industrial applications [2-10]. These properties include a very 
simple process, very cost, and excellent properties of the resultant non-woven nanofiber mat [11-13].

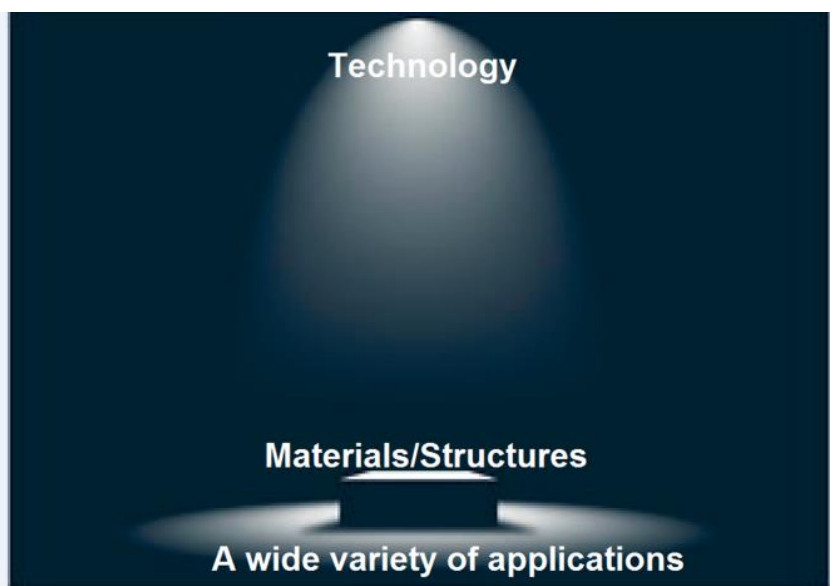

Fig. 2 An innovation in technology always means a series of new materials and structures, which in turn means a wide variety of applications.

In our society, technology innovation is highly desired by all disciplines. Shown in the diagram of Fig. 2, An innovation in technology always means a series of new materials and structures, which in turn means a wide variety of applications. Needless to say, the new biomaterials aimed to provide drug controlled release profiles can be designed and developed by exploiting the advanced electrospinning methods. Meanwhile, these contents should be excellent teaching materials for explaining biomaterial innovations to the undergraduate students in higher school.

\section{EXPLAINING BIOMATERIAL INNOVATIONS RESULTING FROM ELECTROSPUN DRUG-LOADED NANOFIBERS}

How to conceive a new biomaterial when you have the electrospinning system. The students know well about electrospinning and also the fundamental knowledge about drug delivery can answer this question very quickly. In the electrospun nanofibers, the filament-forming polymers often act as matrix to load the functional ingredients. Thus, from a standpoint of the encapsulated active ingredient, different kinds of drug (for different therapeutic effects) can be loaded into the electrospun nanofibers to create new medicated nanoproducts. And based on the most important and difficult issues in pharmaceutics, the students can make it clear that electrospun nanofibers should find fine application if enhancing the dissolution of poorly water-soluble drugs because of the huge surface, small diameter, large porosity and 3-D continuous web structures [14-18].

From a standpoint of the applied polymer matrices, a series of novel biomaterials can be developed with reasonable selection of the drug carriers. For example, when a soluble polymer is selected as the filament-forming matrix, a fast drug release profile ( $\mathrm{C}$ format in Fig. 2); and when an insoluble polymer is exploited, the medicated nanofibers would provide a model-A drug controlled release profile. However, when both soluble and insoluble polymers are formed as the drug carriers within the nanofibers, a model-B biphasic drug controlled release profile can be achieved naturally.

From a standpoint of the electrospinning processes, several different types of electrospinning (single-fluid blending electrospinning, double-fluid coaxial electrospinning, and tri-fluid coaxial electrospinning) can be explored for creating brand-new biomaterials with complicated nanostructures $[19,20]$. Shown in the diagram of Fig. 3, the three electrospinning methods with different number of working fluids can be realized using a similar system (i.e. a spinneret, a power supply, syringe pumps and also a fiber collector). The multiple-fluid will provide more possibilities for tailoring the components, compositions, and spatial distributions of functional ingredients within the complex nanostructures. In turn, these structures will provide more strategies for conceiving nanoproducts furnishing the desired drug controlled release profiles.

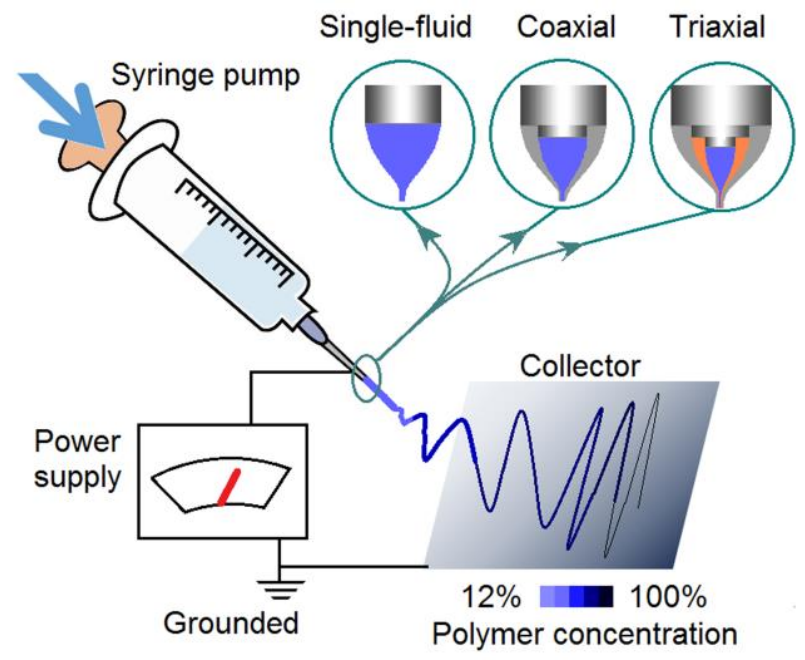

Fig. 3 A diagram about the different types of electrospinning (single-fluid blending electrospinning, double-fluid coaxial electrospinning, and tri-fluid coaxial electrospinning) and the components within an electrospinning system [7].

\section{EXPLAINING BIOMATERIAL INNOVATIONS RESULTING \\ FROM ELECTROSPUN COMPLICATED NANOSTRUCTURES}

During the past over twenty years, the electrospinning processes, and also its counterpart electrospraying, have progressed from the routine single-fluid blending process to the two-fluid coaxial and side-by-side processes, and now is rapidly developing to the three-fluid tri-axial processes and some other complex processes characterized by a combination of side-by-side and coaxial processes [20-28]. Early in 2002, the coaxial EHDA process was reported by Loscertales and his co-worker, which is about a traditional coaxial electrospraying method. Later in 2004, Prof. Dzenis declared that coaxial electrospinning should be one of the most important breakthroughs in the field of electrospinning [29]. In 2015, the even complicated three-fluid coaxial electrospinning was successfully developed and was explored to generate the tri- 
layer nanofibers [7]. Most recently, a complex electrospinning process was reported, in which a side-by-side working fluids acted together as a core part of a coaxial electrospinning [20]. Shown in Fig. 4, this advanced electrospinning technique can not only produce the complicated core-sheath with Janus core structures, but also can fabricate core-sheath and Janus structures in a simple working step.

Needless to say, novel biomaterials with these complicated nanostructures would be more attractive to researchers. Certainly, for the teachers in high school, these electrospun complicated nanostructures should comprise excellent teaching materials to provoke the interests of the students about the related knowledge, the innovation practices, and the development of new biomaterials.

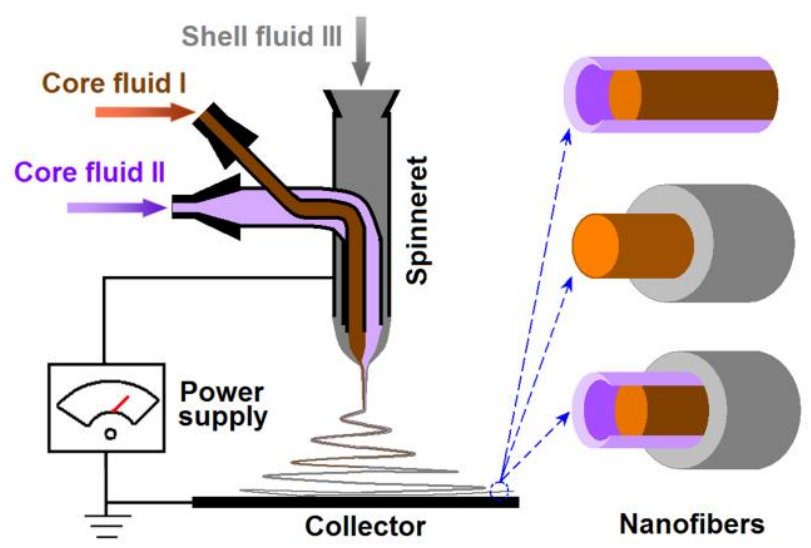

Fig. 4 A simple tri-fluid electrospinning process can produce several complicated nanostructures, including core-sheath, Janus, and core-sheath with Janus core. These complicated nanostructures provide an excellent platform for developing new kinds of biomaterials [20].

\section{EXPLAINING BIOMATERIAL INNOVATIONS RESULTING FROM ELECTROSPRAYING PROCESSES AND ELECTROSPRAYED MEDICATED NANOPARTICLES}

Electrospun nanofibers have a linear morphology, whereas electrosprayed nanoparticles have a round morphology. Linear and circular are the most fundamental shapes in this world. Just as the electrospinning and its nanofibers, electrospraying and its particles can be excellent standpoints for explaining biomaterial innovations to the college students. The electrosprayed nanoparticles can be designed to furnish all the above-mentioned drug controlled release profiles.

Shown in Fig. 5, the electrospraying processes can also be divided into three kinds according to the treated working fluids simultaneously, i.e. the single-fluid blending electrospraying, double-fluid coaxial electrospraying, and trifluid coaxial electrospraying [21-26]. These different electrospraying processes are similar except their spraying heads.

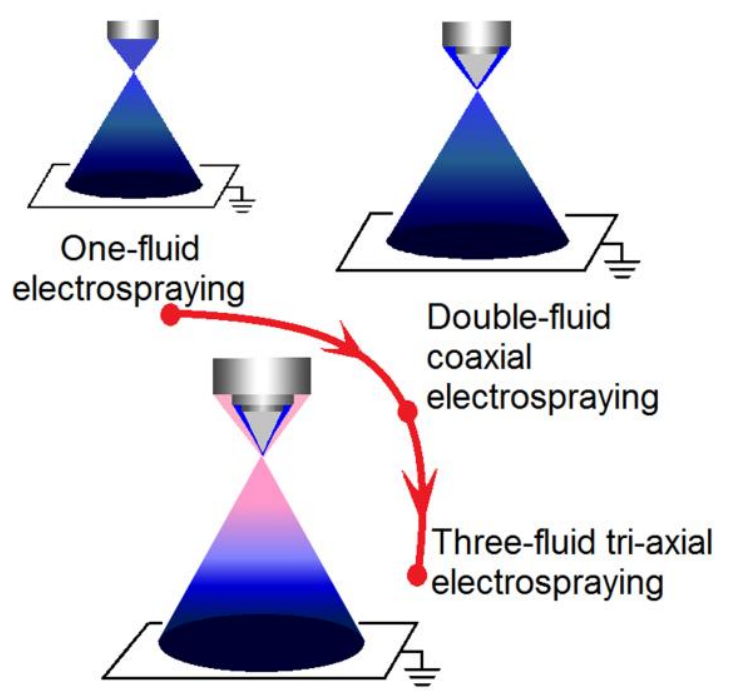

Fig. 5 A schematic about the different types of electrospraying (single-fluid blending electrospraying, double-fluid coaxial electrospraying, and tri-fluid coaxial electrospraying).

Apparently, the electrospun biomaterials providing all types of drug controlled release profiles can give useful hints for both the college students and their teachers. The teachers can exploit the important medicated applications about drug controlled release to explain to the students what, why and how to carry out biomaterial innovations. Shown in Fig. 6, a procedure showing the whole biomaterial innovation processes, from the preparation, to the characterization, and to the final functional applications - colon-targeted pulsatile release of poorly water-soluble drug is demonstrated [26]. This procedure can be similar exploited for designing and developing a wide variety of other biomaterials for furnishing other kinds of drug controlled release profiles.

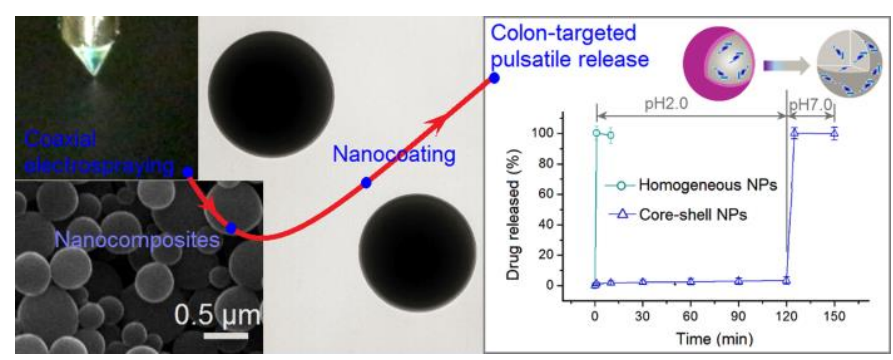

Fig. 6 A procedure shows the whole biomaterial innovation process, from the preparation, to the characterization, and to the final functional applications - colon-targeted pulsatile release of poorly water-soluble drug [26]. 


\section{SUMMARY}

An interesting angle - the medicated applications of biomaterials for providing all kinds of drug controlled release profiles is exhibited for carrying out innovation lessons to the college students majoring material science and engineering. Both innovations about the medicated electrospun nanofibers and also the electrosprayed drug-loaded nanoparticles are clearly explained to the students in a systematic manner. Those related innovations around biomaterials include their generation techniques (all kinds of electrospinning and electrospraying methods) and also the most innovative complex nanostructures such as core-sheath, Janus and coresheath with Janus core. This study paves a new way for the high school teachers to obtain practicable teaching materials from their scientific researches for carrying out innovation lessons to the undergraduate students.

\section{ACKNOWLEDGMENT}

The financial supports from the following projects are appreciated: the Shanghai Education Science Research Project (C17058), the 2017 Graduate curriculum reform project in USST, the National/Shanghai/USST College Student Innovation Project (Nos. grant number: XJ10252324-330, SH10252194 and C201810252044-46-47).

\section{REFERENCES}

[1] D.G. Yu, C. Yang, M. Jin, G.R. Williams, H. Zou, X. Wang, et al, "Medicated Janus fibers fabricated using a Teflon-coated side-by-side spinneret," Colloid. Surface B, vol.138, pp.110-116, Feburary 2016.

[2] Q. Wang, H.P. Li, C. Yang, J.J. Li, and D.G. Yu, "Beads-on-a-string amorphous solid dispersion fabricated using a modified coaxial electrospinning," J. Control. Release, vol.259, pp.e111-e112, 2017.

[3] Y.H. Wu, D.G. Yu, H.P. Li, X.Y. Wu, and X.Y. Li, "Medicated structural PVP/PEG composites fabricated using coaxial electrospinning," e-Polymers, vol.17, pp.39-44, January 2017.

[4] D.G. Yu, H.P. Li, C. Yang, J.J. Li, Q. Wang, and G.R. Williams, "Double-pulsatile release core-shell fibers fabricated using modified tri-axial electrospinning," J. Control. Release, vol.259, pp.e24-e25, 2017.

[5] J.J. Li, Chen Yang, Hai-Peng Li, Qing Wang, and Deng-Guang Yu, "Oral controlled release in accordance with drug adsorption biological rhythm provided by an electrospun structural amorphous solid dispersion," J. Control. Release, vol.259, pp.e61-e62, 2017.

[6] X. Liu, W. Shao, M. Luo, J. Bian, D.G. Yu, "Electrospun blank nanocoating for improved sustained release profiles from medicated gliadin nanofibers," Nanomaterials, vol.8, Article ID 184 (11 pages), 2018.

[7] C. Yang, D.G. Yu, D. Pan, X.K. Liu, X. Wang, S.W.A. Bligh, et al, "Electrospun $\mathrm{pH}$-sensitive core-shell polymer nanocomposites fabricated using a tri-axial processes," Acta Biomater., vol.35, pp.77-86, April 2016.

[8] G.Z. Yang, H.P. Li, J.H. Yang, J. Wan, and D.G. Yu, "Influence of working temperature on the formation of electrospun polymer nanofibers," Nanoscale Res. Lett., vol.12, Article 15, January 2017.

[9] Y.Y. Yang, Z.P. Liu, D.G. Yu, K. Wang, P. Liu, X. Chen, "Colonspecific pulsatile drug release provided by electrospun shellac nanocoating on hydrophilic amorphous composites," Int. J. Nanomed., vol.2018, pp. 2395-2404, 2018.

[10] Q. Wang, D.G. Yu, L.L. Zhang, X.K. Liu, Y.C. Deng, and M. Zhao, "Electrospun hypromellose-based hydrophilic composites for rapid dissolution of poorly water-soluble drug," Carbohydr. Polym., vol.174, pp.617-625, Oct, 2017.

[11] Y.H. WU, C. Yang, X.Y. Li, J.Y. Zhu, and D.G. Yu, "Medicated nanofibers fabricated using $\mathrm{NaCl}$ solutions as shell fluids in a modified coaxial electrospinning," J. Nanomater., vol.2016, Article ID 8970213, 2016.

[12] K. Wang, X.K. Liu, X.H. Chen, D.G. Yu, Y.Y. Yang, and P. Liu, "Electrospun hydrophilic Janus nanocomposites for the rapid onset of therapeutic action of helicid," ACS Appl. Mater. Interfaces, vol.10, pp.2859-2867, 2018.

[13] G.Z. Yang, J.J. Li, D.G. Yu, M.F. He, J.H. Yang, and G.R. Williams, "Nanosized sustained-release drug depots fabricated using modified triaxial electrospinning," Acta Biomater., vol.53, pp.233-241, April 2017.

[14] Y. Xu, J.J. Li, D.G. Yu, G.R. Williams, J.H. Yang, and X. Wang, "Influence of the drug distribution in electrospun gliadin fibers on drugrelease behavior," Eur. J. Pharm. Sci., vol.106, pp.422-430, August 2017.

[15] Z. Zhang, W. Li, G. Wang, Y.L. Qu, and D.G. Yu, "Electrospun 4th generation solid dispersions of poorly water-soluble drug utilizing two different processes," J. Nanomater. Vol.2018, Article ID 2012140, 2018.

[16] Q. Wang, D.G. Yu, S.Y. Zhou, C. Li, and M. Zhao, "Fabrication of amorphous electrospun medicated-nanocomposites using a Teflonbased concentric spinneret," e-Polymer, vol.18, pp.3-11, 2018.

[17] Y.H. Wu, D.G. Yu, J.J. Li, Q. Wang, H.P. Li, and X.Y. Li, "Medicated multiple-component polymeric nanocomposites fabricated using electrospraying," Polym. Polym. Compos., vol.25, pp.57-62, 2017.

[18] M. Jin, D.G. Yu, X. Wang, C.F.G.C. Geraldes, G.R. Williams, and S.W.A. Bligh, "Electrospun contrast agent-loaded fibers for colontargeted MRI," Adv. Healthcare Mater., vol.5, pp.977-985, April 2016.

[19] Y.H. WU, H.P. Li, X.X. Shi, J. Wan, Y.F. Liu, and D.G. Yu, "Effective utilization of the electrostatic repulsion for improved alignment of electrospun nanofibers," J. Nanomater., vol.2016, Article ID2067383, 2016.

[20] D.G. Yu, J.J. Li, M. Zhang, and G.R. Williams, "High-quality Janus nanofibers prepared using three-fluid electrospinning," Chem. Commun., vol.53, pp.4542-4545, April 2017.

[21] X.Y. Li, Z.B. Zheng, D.G. Yu, X.K. Liu, Y.L. Qu, and H.L. Li, "Electrosprayed sperical ethylcellulose nanoparticles for an improved sustained-release profile of anticancer drug," Cellulose, vol.24, pp.5551-5564, 2017.

[22] Z.P. Liu, Y.Y. Zhang, D.G. Yu, D. Wu, and H.L. Li, "Fabrication of sustained-release zein nanoparticles via modified coaxial electrospraying," Chem. Eng. J., vol.334, pp.807-816, 2018.

[23] Y.Y. Yang, M. Zhang, Z.P. Liu, K. Wang, and D.G. Yu, "Meletin sustained-release gliadin nanoparticles prepared via solvent surface modification on blending electrospraying," App. Surf. Sci., vol.434, pp.1040-1047, 2018.

[24] K. Wang, H.F. Wen, D.G. Yu, Y. Yang, and D.F. Zhang, "Electrosprayed hydrophilic nanocomposites coated with shellac for colon-specific delayed drug delivery," Mater. Design, vol.143, pp.248255, 2018.

[25] Z.P. Liu, L.L. Zhang, Y.Y. Yang, D. Wu,G. Jiang, and D.G. Yu, "Preparing composite nanoparticles for immediate drug release by modifying electrohydrodynamic interfaces during electrospraying," Powder Technol., vol.327, pp.179-187, 2018.

[26] Y.Y. Yang, M. Zhang, K. Wang, and D.G. Yu. "pH-sensitive polymer nanocoating on hydrophilic composites fabricated using modified coaxial electrospraying," Mater. Lett., vol.227, pp. 93-96, 2018.

[27] H.P. Li, L.L. Zhang, Y.Y. Zhang, and D.G. Yu, "Core-shell medicated nanoparticles prepared using coaxial electrospray for fast dissolution of paracetamol," J. Invest. Med., vol.65 (Suppl 7), A2, 2017, DOI: 10.1136/jim-2017-MEBabstracts.5.

[28] M. Jin, D.G. Yu, C.F.G.C. Geraldes, G.R. Williams, S.W.A. Bligh, "Theranostic fibers for simultaneous imaging and drug delivery," Mol. Pharm., vol.13, pp. 2457-2465, 2016.

[29] Y. Dzenis, "Spinning continuous fibers for nanotechnology," Science vol. 304, pp.1917-1919, 2004. 